\title{
Correlates of stunting among under-five children in Bangladesh: a multilevel approach
}

\author{
Papia Sultana ${ }^{1 *}$, Md. Mahfuzur Rahman² and Jahanara Akter $^{2}$
}

\begin{abstract}
Background: Child malnutrition still remains a major cause of childhood morbidity and mortality in Bangladesh. This study aims to determine the prevalence and identify the associated risk factors of child malnutrition in Bangladesh using multilevel logistic regression model on data from the Bangladesh Demographic and Health Survey (BDHS), 2014.

Methods: A total sample of 6965 children aged 0-59 months was extracted from BDHS 2014. We performed descriptive analysis and multilevel generalized linear regression analysis with clustered data structure.

Results: Our findings show that among children the prevalence of moderate and severe values was respectively: 25 and 12\% for stunting; 11 and 3.1\% for wasting; 25 and $7.9 \%$ for underweight. The probability of stunting increased with age, with highest rate among children aged 36-47 months, which was significantly higher than children aged less than 6 months $(\mathrm{OR}=6.71,95 \% \mathrm{Cl}=4.46,10.10)$. Female children are found to be $11 \%$ less likely to be stunted than male children $(\mathrm{OR}=0.89,95 \% \mathrm{Cl}=0.78,1.02)$. Children with birth interval less than 24 months were significantly more likely to be stunted than children of first birth by $36 \%(\mathrm{OR}=1.36,95 \% \mathrm{Cl}=1.11,1.67)$. Mothers with a normal BMI were $16 \%$ less likely to have children with stunting compared to mothers who are underweight $(\mathrm{OR}=0.84,95 \% \mathrm{Cl}=0.76,0.93)$. Other factors which were associated with a higher risk of stunting included parents with lower educational levels, children from the poorest wealth index, and mothers aged less than 20 years as first birth.

Conclusion: Government and non-government organization should generate effective program to aware women of reproductive age about adverse effect of short birth interval, and to aware parents about standard height and weight according to age and gender of children. Overall, necessary steps may be taken to make people educated and to reduce household wealth inequality to improve nutritional status of children.
\end{abstract}

Keywords: Child malnutrition, Stunting, Prevalence, Multilevel generalized linear regression, Odds ratio, Bangladesh

\section{Background}

Malnutrition is defined by the World Health Organization as any deficiencies, excesses, or imbalances in a person's intake of energy and/or nutrients [1]. Child nutrition status is an imperative indicator of poverty in a population; and poverty, malnutrition and disease are intertwined each other [2,3]. Malnutrition has been referred as the single greatest threat to the world's public health, especially for the developing countries, by the

\footnotetext{
* Correspondence: papia.stat@ru.ac.bd

${ }^{1}$ Department of Statistics, University of Rajshahi, Rajshahi, Bangladesh

Full list of author information is available at the end of the article
}

World Health Organization [1]. The nutritional status of under-five children is the most sensitive indicator of a society, country as well as world public health status. Malnutrition is an underlying cause of $45 \%$ of child death- accounting for almost one-half of the global total of children's deaths [4]. Rates of child malnutrition in Bangladesh are among the highest in the world, with rates of stunting affecting more than $54 \%$ of preschool-age children, underweight in 56\% and wasting in 17\% [5]. In Bangladesh, nutrition-attributable mortality rate in children is about 53 per 1000 live birth according to Bangladesh Demographic

(c) The Author(s). 2019 Open Access This article is distributed under the terms of the Creative Commons Attribution 4.0 International License (http://creativecommons.org/licenses/by/4.0/), which permits unrestricted use, distribution, and 
and Health Survey 2011 [6]. Survivors are left vulnerable to illnesses, stunted growth and intellectual impairment. The prevalence of stunting in children has been steadily decreasing, from $51 \%$ in 2004 to $37 \%$ in 2014 [7, 8]. However, the average annual rate of reduction (AARR) of stunting in Bangladesh is 2.7 which is much less than the required 3.9 AARR [9] to reach the global World Health Assembly (WHA) target to have $40 \%$ reduction in child malnutrition by 2025 [10]. At current rate, Bangladesh is not likely to achieve the WHA target for reducing childhood malnutrition.

To further enable decline of malnutrition rate in children and reach zero hunger goals as set by international targets, comprehensive preventive policies targeting evidence-based high-risk groups is crucial. Numerous studies have focused on child malnutrition in Bangladesh and/or it's associated risk factors [11-39]. However, in studies assessing risk factors, most utilized standard logistic regression as the statistical model; few have used multivariate approach such as one study using Bangladesh Demographic and Health Survey (BDHS) data from 2011 [40], and multilevel analysis using BDHS data from 2007 [11]. This study clearly demonstrated the significant variation of child malnutrition to hierarchical factors such as residence and division. Therefore, to determine child malnutrition in correct level, the variation due to hierarchical factors should be adjusted in the model through method of multilevel analysis [41-44]. To fill this gap in literature using the most recent datasets, our study aimed to identify the significant risk factors of stunting among under-five children in Bangladesh using multilevel generalized linear regression model using BDHS 2014 data.

\section{Methods}

\section{Sample selection}

The data used for the present study has been derived from BDHS, 2014, a nationally-representative survey conducted by the National Institute for Population Research and Training (NIPORT) of the Ministry of Health and Family Welfare [45]. The 2014 BDHS collected data on the nutritional status of children by measuring the height and weight of all children aged between 0 and 59 months in the selected households. The sample for the BDHS-2014 is nationally representative and covers the entire population residing in non-institutional dwelling units in the country. The survey used a sampling frame from the list of enumeration areas (EAs) of the 2011 Population and Housing Census of the People's Republic of Bangladesh, provided by the Bangladesh Bureau of Statistics (BBS). The primary sampling unit (PSU) for the survey is an EA created to have an average of about 120 households. With the design, the survey selected 18,000 residential households, which were expected to result in completed interviews with about 18,000 ever-married women of age $15-49$ years. A total of 17,863 ever-married women of age 15-49 years were interviewed, for a response rate of $98 \%$ (Fig. 1). Details about the survey collection method can be found in literature [46]. For the current study, the data has been screened for the under-five children for whom height and weight are available (Fig. 2). Therefore, the study uses a sample of size 6965 extracted from 7886 of BDHS 2014 data.

Three standard indices of physical growth that describe the nutritional status of children are: (i) Heightfor-age, (ii) Weight-for-height, and (iii) Weight-for-age. Each of these indices provides different information about growth and body composition that can be used to assess nutritional status of under-five children. The nutritional status of children in the survey population is compared with the World Health Organization (WHO) Child Growth Standards to create z-scores [46] resulting the indicators as stunting, wasting and underweight. Standard deviations below the reference median of the WHO reference population in terms of height-for-age was defined as stunted ( $-2 \mathrm{SD})$ or severely stunted (-3 SD). Similarly, standard deviations below the reference median of the WHO reference population in terms of weight-for-age was defined as wasted $(-2 \mathrm{SD})$ or severely wasted $(-3 \mathrm{SD})$; standard deviations below the reference median of the WHO reference population in terms of weight-for-height was defined as underweight $(-2 \mathrm{SD})$ or severely underweight $(-3 \mathrm{SD})$. However, zscores computed for the three health indicators heightfor-age, weight-for-height, and weight-for-age were used in this study.

Size of child at birth or a child's birth weight was recorded based on mother's perception [46]. A child's birth weight less than $2.5 \mathrm{~kg}$ was considered as less than average, and lager than 2.5 was considered as larger than average. Mother's BMI was classified as underweight if it was less than $18.5 \mathrm{~kg} / \mathrm{m}^{2}$, normal if it was between $18.5-$ $24.9 \mathrm{~kg} / \mathrm{m}^{2}$, overweight if it was between 25 and $29.9 \mathrm{~kg} /$ $\mathrm{m}^{2}$, and obese if it was above $30 \mathrm{~kg} / \mathrm{m}^{2}$. For the educational level, primary level was defined as completing grade 5 , secondary level was defined as completing grade 10. The wealth index was created in three steps. In the first step, a subset of household indicators (for example, access to safe drinking water, sanitation facilities, structure of housing, crowdedness of housing, type of fuel used for cooking, household possessions etc.) common to urban and rural areas was used to create wealth scores for households in both areas. Categorical variables were transformed into separate dichotomous $(0-1)$ indicators. Those indicators and those that were continuous 
The sampling frame was Population Census of the People's Republic of Bangladesh conducted in 2011

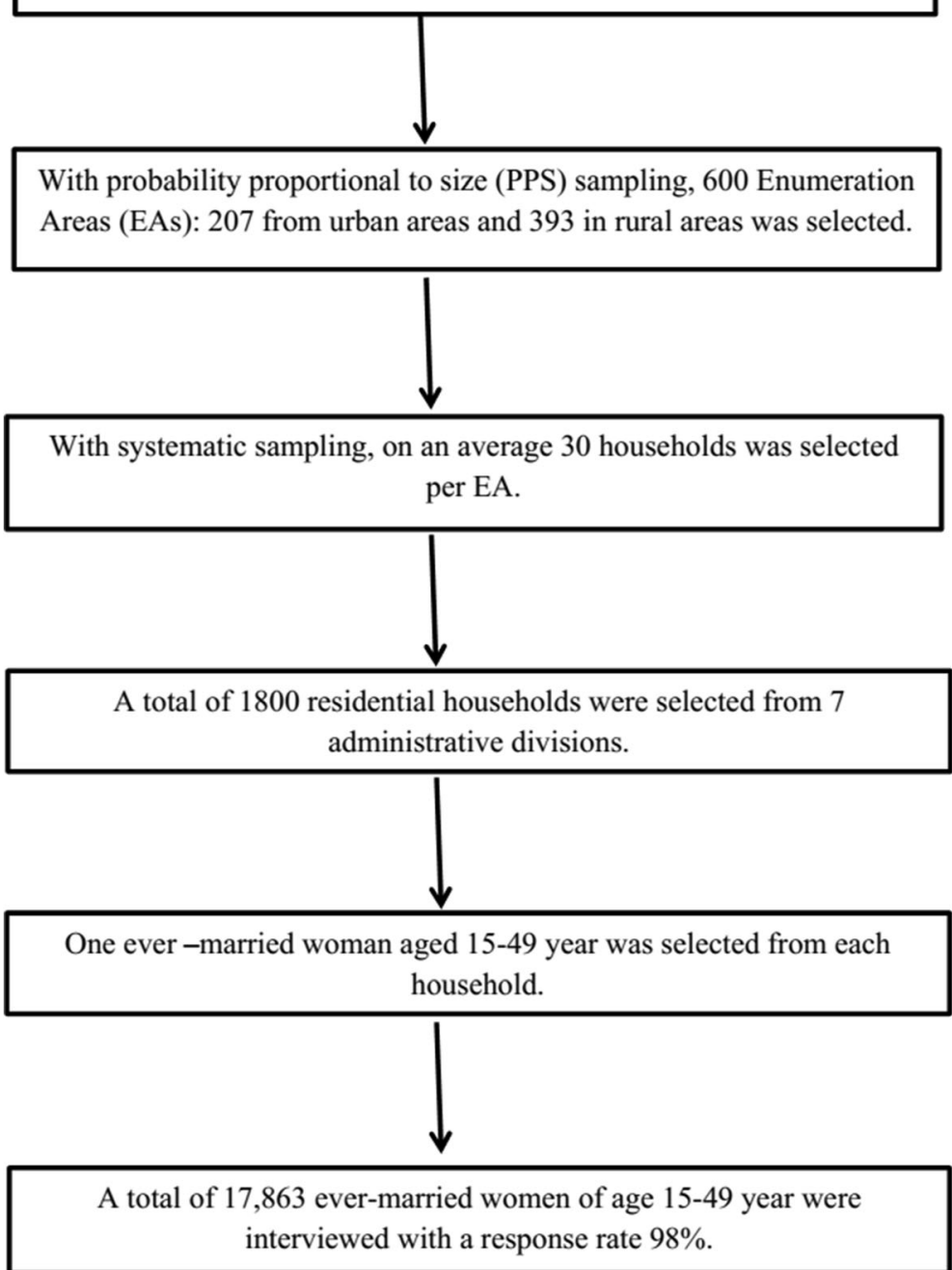

Fig. 1 Study design of Bangladesh Demographic Health Survey, 2014

were then examined using a principal components analysis to produce a common factor score for each household. In the second step, separate factor scores were produced for households in urban and rural areas using area specific indicators. The third step combined the separate area-specific factor scores to produce a nationally applicable combined wealth index by adjusting areaspecific scores through a regression on the common factor scores. The resulting combined wealth index had a mean of zero and a standard deviation of one. Once the index was computed, national-level wealth quintiles (from lowest to highest) were obtained by assigning the household score to each de jure household member, ranking each person in the population by his or her score, and then dividing the ranking into five equal categories, each comprising $20 \%$ of the population.
Computations for wealth index were done by the authority of the survey and details can be found in survey literature [46]. In this study, stunting was used as the indicator of nutritional status of under-five children and used as the response variable in generalized linear regression analysis.

\section{Statistical analysis}

We performed descriptive analysis to determine the characteristics of the study participants, which were presented with the frequency and percentage. Then, bivariate analyses were performed to compare child malnutrition (stunting, wasting and underweight) to the confounding variable. $P$-values had been obtained from bivariate simple linear regression to $\mathrm{z}$-scores to check the association sex residence and division with child 
Ever-married women of age 15-49 year in Bangladesh

Demographic Health Survey, 2014.

$(\mathrm{N}=17,863)$

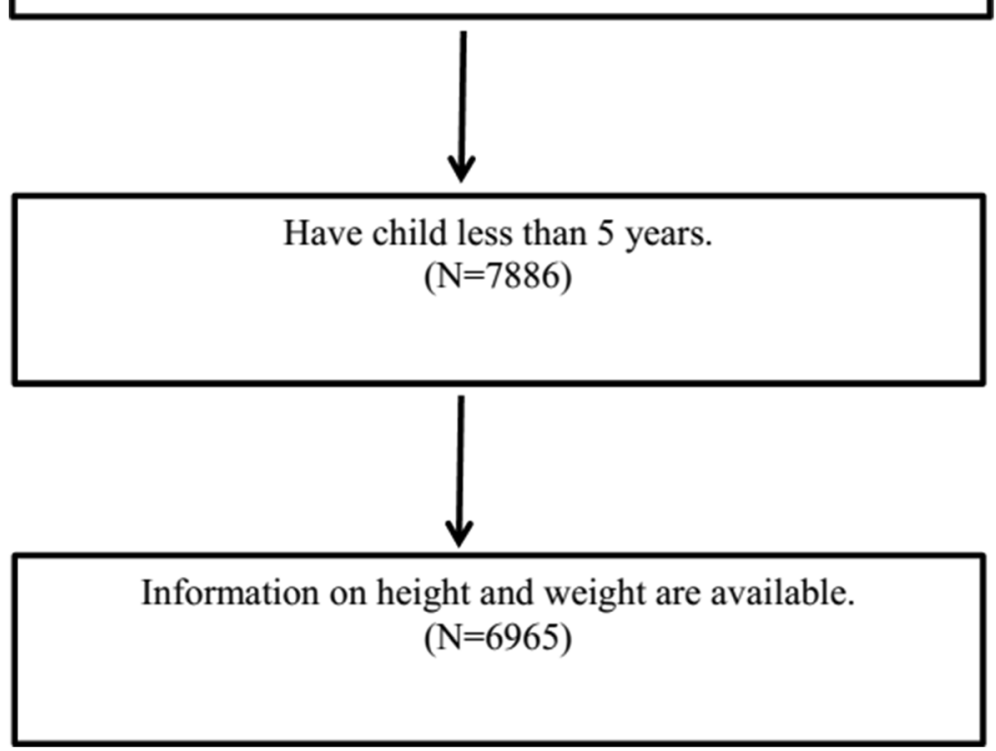

Fig. 2 Data screening for under-five children in Bangladesh

malnutrition. Lastly, multilevel generalized linear regression analysis had been performed with logit link and binomial family to identify significantly associated risk factors of child malnutrition. Various socio-demographic and economic variables were considered as risk factors. Division and residence of the respondents have been considered as the hierarchical variables. Two-level logistic regression (model-1 and model-2) and three level (model-3) logistic regression have been performed. In model-1 (two level) division has been considered as second level sources of variation; in model-2 (two level) residence has been considered as second level sources of variation; and in model-3 (three level) division has been considered as second level and residence has been as third level sources of variation. Statistical software StataSE version 13 (Stata Corp, USA) has been used to carry out statistical analyses. Missing values had been avoided in advanced analysis.

\section{Results}

A total of 6965 children aged 0-59 months had been included in the study, of whom 3571 (51.3\%) were male and 3394 (48.7\%) were female (Table 1). A significant percentage (19.1\%) of children was below average size at birth. Among the mothers $73 \%$ gave their first birth at before age 20 years and $22.4 \%$ mothers were underweighted. About $20.9 \%$ mothers had no antenatal visits during pregnancy and $15.4 \%$ mothers had no formal education. Among the respondents $68.6 \%$ were from rural area and $21.7 \%$ were of poorest wealth index.

The data showed that among the children $12.2 \%$ were severely stunted, $24.9 \%$ were moderately stunted and $62.9 \%$ were well nourished (Table 2). It was found that $3.2 \%$ children were severely wasted and $11.7 \%$ were moderately wasted. About $8.3 \%$ of the children were found to be severely underweight, while $24.7 \%$ were moderately underweight.

From Table 3, it had been found that stunting and underweight differ significantly to residence and division. It had been also found that rural children are more stunted than urban children and children of Sylhet division are mostly stunted. However, wasting differs significantly to gender, residence and division.

Table 4 represents odds ratio (OR) with 95\% confidence interval (CI) obtained from multilevel generalized linear regression with binomial family and logit link to nutritional status of under-five children. From the results of Akaike Information Criterion (AIC), Bayesian Information Criterion (BIC), Intraclass Correlation Coefficient (ICC) and Median Odds Ratio (MOR), Model-III was the best. Our results showed that the odds of being stunting significantly increased with age, with highest $\mathrm{OR}$ in $36-47$ months age group $(\mathrm{OR}=6.71,95 \% \mathrm{CI}=$ $4.56,10.10)$. Children of preceding birth interval of less than 24 months was also significantly associated with increased odds of stunting $(\mathrm{OR}=1.36,95 \% \mathrm{CI}=1.11$, 1.67). Conversely, female children was found to be $11 \%$ 
Table 1 Characteristics of the study subjects, BDHS-2014

\begin{tabular}{|c|c|c|c|}
\hline \multirow[t]{2}{*}{ Characteristic } & \multirow{2}{*}{$\begin{array}{l}\text { Total (sample size = } \\
6965) \\
\text { Frequency(Percentage) }\end{array}$} & Male (sample size $=3571$ ) & Female $($ sample size $=3394)$ \\
\hline & & Frequency(Percentage) & Frequency(Percentage) \\
\hline \multicolumn{4}{|l|}{ Age in months } \\
\hline mean & 29.56 & 29.42 & 29.72 \\
\hline$<6$ & $558(8.01)$ & $302(8.46)$ & $256(7.54)$ \\
\hline $6-11$ & $786(11.28)$ & $396(11.09)$ & $390(11.49)$ \\
\hline $12-23$ & $1456(20.90)$ & $744(20.83)$ & $712(20.98)$ \\
\hline $24-35$ & $1405(20.17)$ & 719 (20.13) & $686(20.21)$ \\
\hline $36-47$ & $1377(19.77)$ & 701 (19.63) & $676(19.92)$ \\
\hline $48-59$ & $1383(19.86)$ & 709 (19.85) & $674(19.86)$ \\
\hline \multicolumn{4}{|l|}{ Size of child at birth } \\
\hline Very large & $96(2.28)$ & $55(2.55)$ & $41(2.01)$ \\
\hline Larger than average & $456(10.84)$ & $251(11.61)$ & $205(10.03)$ \\
\hline Average & $2849(67.75)$ & $1466(67.84)$ & $1383(67.66)$ \\
\hline Smaller than average & $544(12.94)$ & $271(12.54)$ & 273 (13.36) \\
\hline Very small & $260(6.18)$ & $118(5.46)$ & $142(6.95)$ \\
\hline \multicolumn{4}{|l|}{$\begin{array}{l}\text { Live birth } \\
\text { between births }\end{array}$} \\
\hline Yes & $4(0.09)$ & $2(0.09)$ & $2(0.10)$ \\
\hline No & $4261(99.91)$ & $2204(99.91)$ & $2057(99.90)$ \\
\hline \multicolumn{4}{|l|}{$\begin{array}{l}\text { Preceding birth } \\
\text { interval (months) }\end{array}$} \\
\hline First birth & $2700(38.84)$ & $1365(38.31)$ & $1335(39.40)$ \\
\hline$<24$ & $476(6.85)$ & $243(6.82)$ & $233(6.88)$ \\
\hline $24-47$ & $1388(19.97)$ & $733(20.57)$ & 655 (19.33) \\
\hline $48+$ & $2387(34.34)$ & $1222(34.30)$ & $1165(34.39)$ \\
\hline \multicolumn{4}{|l|}{$\begin{array}{l}\text { Age of mother at } \\
1 \text { st birth (years) }\end{array}$} \\
\hline$<20$ & $5085(73.01)$ & $2608(73.03)$ & $2477(72.98)$ \\
\hline $20-30$ & $1838(26.39)$ & $940(26.32)$ & $898(26.46)$ \\
\hline $30+$ & $42(0.60)$ & $23(0.64)$ & $19(0.56)$ \\
\hline \multicolumn{4}{|l|}{ Mother's BMI } \\
\hline Underweight & $1556(22.42)$ & $797(22.41)$ & 759 (22.44) \\
\hline Normal & $4067(58.60)$ & $2096(58.93)$ & $1971(58.26)$ \\
\hline Overweight & $1097(15.81)$ & $543(15.27)$ & $554(16.38)$ \\
\hline Obese & $220(3.17)$ & $121(3.40)$ & $99(2.93)$ \\
\hline \multicolumn{4}{|l|}{$\begin{array}{l}\text { Frequency of antenatal } \\
\text { visits during pregnancy }\end{array}$} \\
\hline No antenatal care visits & $848(20.95)$ & $434(20.80)$ & $414(21.11)$ \\
\hline Antenatal visits & $3196(78.95)$ & $1651(79.11)$ & $1545(78.79)$ \\
\hline Don't know & $4(0.10)$ & $2(0.10)$ & $2(0.10)$ \\
\hline \multicolumn{4}{|l|}{ Duration of breastfeeding } \\
\hline Ever Breastfed & $535(13.15)$ & $275(13.13)$ & $260(13.18)$ \\
\hline Never breastfed & $46(1.13)$ & $22(1.05)$ & $24(1.22)$ \\
\hline Still breastfeeding & 3481 (85.59) & $1798(85.82)$ & $1683(85.34)$ \\
\hline Don't know & $5(0.12)$ & $0(0.00)$ & $5(0.25)$ \\
\hline \multicolumn{4}{|l|}{ Mother's educational level } \\
\hline No education & 1076 (15.45) & $588(16.47)$ & 488 (14.38) \\
\hline
\end{tabular}


Table 1 Characteristics of the study subjects, BDHS-2014 (Continued)

\begin{tabular}{|c|c|c|c|}
\hline \multirow[t]{2}{*}{ Characteristic } & \multirow{2}{*}{$\begin{array}{l}\text { Total (sample size }= \\
6965) \\
\text { Frequency(Percentage) }\end{array}$} & Male (sample size $=3571$ ) & \multirow{2}{*}{$\begin{array}{l}\text { Female }(\text { sample size }=3394) \\
\text { Frequency }(\text { Percentage })\end{array}$} \\
\hline & & Frequency(Percentage) & \\
\hline Primary & $1934(27.77)$ & $957(26.80)$ & 977 (28.79) \\
\hline Secondary & 3219 (46.22) & $1631(45.67)$ & $1588(46.79)$ \\
\hline Higher & $736(10.57)$ & $395(11.06)$ & $341(10.05)$ \\
\hline \multicolumn{4}{|l|}{ Mother's employment status } \\
\hline Yes & $1747(25.09)$ & 885 (24.79) & $862(25.40)$ \\
\hline No & $5217(74.91)$ & $2685(75.21)$ & $2532(74.60)$ \\
\hline \multicolumn{4}{|l|}{ Father's educational level } \\
\hline No education & $1736(24.93)$ & $875(24.50)$ & $861(25.38)$ \\
\hline Primary & $2100(30.16)$ & $1047(29.32)$ & $1053(31.04)$ \\
\hline Secondary & $2118(30.42)$ & $1119(31.34)$ & 999 (29.45) \\
\hline Higher & $1009(14.49)$ & $530(14.84)$ & $479(14.12)$ \\
\hline \multicolumn{4}{|l|}{ Father's occupation } \\
\hline Professional & $651(9.38)$ & $335(9.40)$ & $316(9.35)$ \\
\hline Sales & $1384(19.94)$ & 705 (19.79) & $679(20.09)$ \\
\hline Agricultural-self employ & $642(9.25)$ & $346(9.71)$ & $296(8.76)$ \\
\hline Agricultural-employ & $1046(15.07)$ & $527(14.80)$ & $519(15.36)$ \\
\hline Services & $583(8.40)$ & $272(7.64)$ & $311(9.20)$ \\
\hline Skilled manual & $2460(35.44)$ & $1280(35.93)$ & $1180(34.91)$ \\
\hline Household and domestic & $132(1.90)$ & $71(1.99)$ & $61(1.80)$ \\
\hline Don't work & $44(0.63)$ & $26(0.73)$ & $18(0.53)$ \\
\hline \multicolumn{4}{|l|}{ Division } \\
\hline Barisal & $812(11.66)$ & $416(11.65)$ & $396(11.67)$ \\
\hline Chittagong & $1320(18.95)$ & $667(18.68)$ & $653(19.27)$ \\
\hline Dhaka & $1213(17.42)$ & $620(17.36)$ & $593(17.47)$ \\
\hline Khulna & $774(11.11)$ & $399(11.17)$ & $375(11.05)$ \\
\hline Rajshahi & $875(12.56)$ & $442(12.38)$ & $433(12.76)$ \\
\hline Rangpur & $865(12.42)$ & $456(12.77)$ & 409 (12.05) \\
\hline Sylhet & $1106(15.88)$ & $571(15.99)$ & $535(15.76)$ \\
\hline \multicolumn{4}{|l|}{ Type of Place of residence } \\
\hline Urban & $2188(31.41)$ & $1129(31.62)$ & $1059(31.20)$ \\
\hline Rural & $4777(68.59)$ & $2442(68.38)$ & $2335(68.80)$ \\
\hline \multicolumn{4}{|l|}{ Wealth index } \\
\hline Poorest & $1515(21.75)$ & $794(22.23)$ & $721(21.24)$ \\
\hline Poorer & $1307(18.77)$ & $674(18.87)$ & $633(18.65)$ \\
\hline Middle & $1379(19.80)$ & $677(18.96)$ & $702(20.68)$ \\
\hline Richer & $1420(20.39)$ & $732(20.50)$ & $688(20.27)$ \\
\hline Richest & $1344(19.30)$ & $694(19.43)$ & $650(19.15)$ \\
\hline
\end{tabular}

Table 2 Prevalence of stunting, wasting and underweight of under-five children in Bangladesh, BDHS-2014

\begin{tabular}{llll}
\hline Category & $\begin{array}{l}\text { Stunting (Height for age) Frequency } \\
(\%)\end{array}$ & $\begin{array}{l}\text { Wasting (Weight for height) Frequency } \\
(\%)\end{array}$ & $\begin{array}{l}\text { Underweight (Weight for age) Frequency } \\
(\%)\end{array}$ \\
\hline Severely malnourished & $833(11.96)$ & $213(3.06)$ & $552(7.93)$ \\
$\begin{array}{l}\text { Moderately } \\
\text { malnourished }\end{array}$ & $1714(24.61)$ & $792(11.37)$ & $1708(24.52)$ \\
Normal (well-nourished) & $4418(63.43)$ & $5960(85.57)$ & $4705(67.55)$ \\
\hline
\end{tabular}




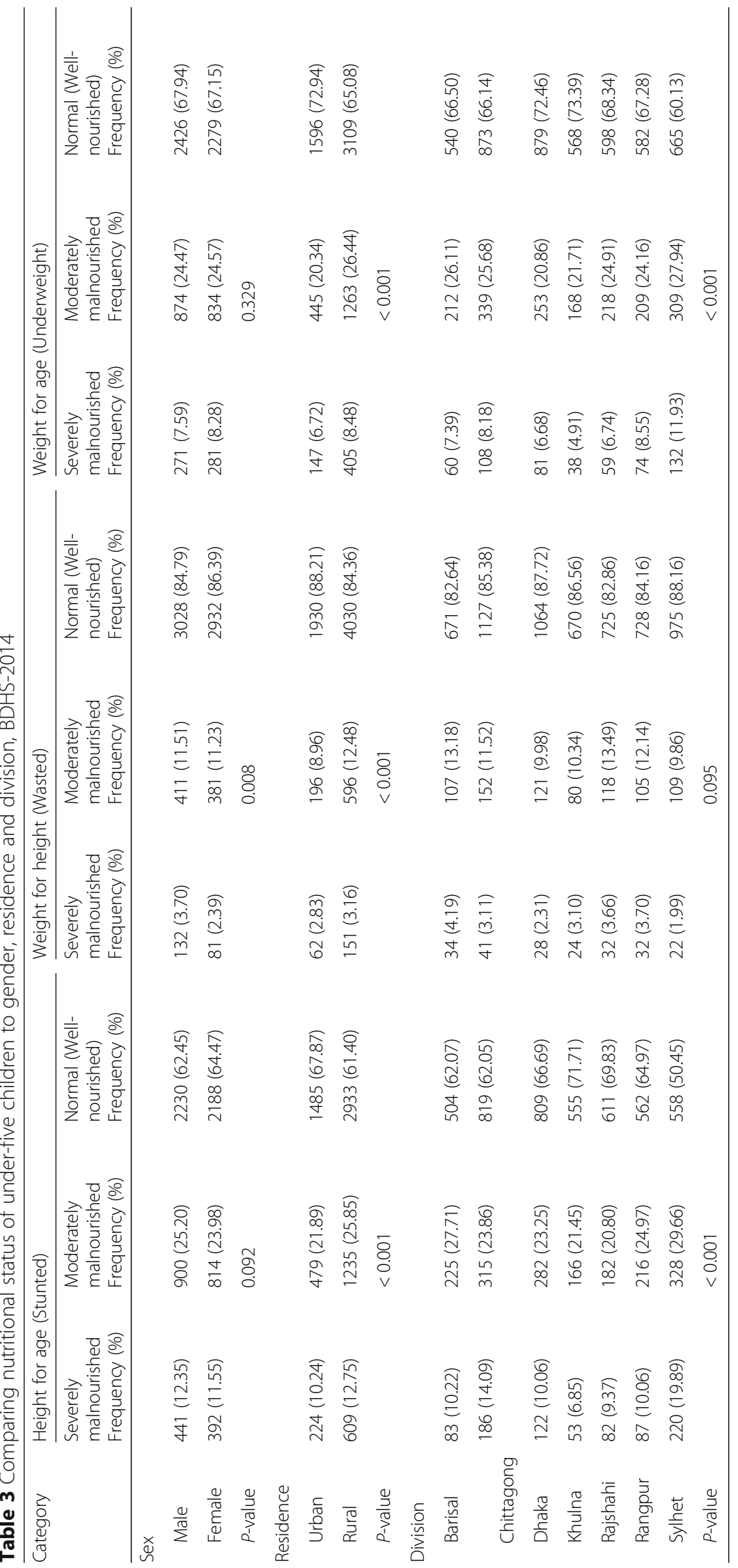


Table 4 Odds Ratio from multilevel generalized linear regression to stunting status of children in Bangladesh, BDHS-2014

\begin{tabular}{|c|c|c|c|}
\hline \multirow[t]{2}{*}{ Variable } & \multicolumn{3}{|c|}{ Odds Ratio (95\% Confidence Interval) } \\
\hline & Model $1^{\text {a }}$ & Model $2^{b}$ & Model $3^{c}$ \\
\hline \multicolumn{4}{|l|}{ Age in months } \\
\hline$<6(\mathrm{RC})$ & 1.00 & 1.00 & 1.00 \\
\hline $6-11$ & $1.94(1.27-2.97)$ & $1.89(1.52-2.35)$ & $1.94(1.27-2.96)$ \\
\hline $12-23$ & $5.08(3.75-6.90)$ & $4.91(3.82-6.32)$ & $5.09(3.76-6.88)$ \\
\hline $24-35$ & $5.87(4.15-8.28)$ & $5.72(5.44-6.02)$ & $5.88(4.16-8.31)$ \\
\hline $36-47$ & $6.71(4.47-10.07)$ & $6.55(5.96-7.20)$ & $6.71(4.46-10.10)$ \\
\hline $48-59$ & $4.47(3.07-6.51)$ & $4.40(4.33-4.47)$ & $4.48(3.08-6.53)$ \\
\hline \multicolumn{4}{|l|}{ Sex of child } \\
\hline Male (RC) & 1.00 & 1.00 & 1.00 \\
\hline Female & $0.89(0.78-1.02)$ & $0.89(0.85-0.93)$ & $0.89(0.78-1.02)$ \\
\hline \multicolumn{4}{|l|}{$\begin{array}{l}\text { Preceding birth } \\
\text { interval (months) }\end{array}$} \\
\hline First birth (RC) & 1.00 & 1.00 & 1.00 \\
\hline$<24$ & $1.36(1.11-1.67)$ & $1.47(1.22-1.77)$ & $1.36(1.11-1.67)$ \\
\hline $24-47$ & $1.15(0.96-1.39)$ & $1.25(1.14-1.38)$ & $1.16(0.96-1.40)$ \\
\hline $48+$ & $0.88(0.77-1.02)$ & $0.88(0.83-0.93)$ & $0.89(0.77-1.02)$ \\
\hline \multicolumn{4}{|l|}{$\begin{array}{l}\text { Age of mothers at } \\
1 \text { st birth (years) }\end{array}$} \\
\hline$<20(\mathrm{RC})$ & 1.00 & 1.00 & 1.00 \\
\hline $20-30$ & $0.87(0.77-0.98)$ & $0.90(0.77-1.06)$ & $0.87(0.76-0.98)$ \\
\hline $31+$ & $0.88(0.48-1.59)$ & $0.90(0.53-1.53)$ & $0.87(0.49-1.56)$ \\
\hline \multicolumn{4}{|l|}{ Mother's BMI } \\
\hline Underweight (RC) & 1.00 & 1.00 & 1.00 \\
\hline Normal & $0.84(0.77-0.93)$ & $0.83(0.78-0.89)$ & $0.84(0.76-0.93)$ \\
\hline Overweight & $0.62(0.51-0.76)$ & $0.60(0.54-0.67)$ & $0.62(0.51-0.76)$ \\
\hline Obese & $0.65(0.46-0.93)$ & $0.60(0.49-0.73)$ & $0.65(0.46-0.92)$ \\
\hline \multicolumn{4}{|l|}{ Mother's educational level } \\
\hline No education (RC) & 1.00 & 1.00 & 1.00 \\
\hline Primary & $0.96(0.73-1.26)$ & $0.93(0.90-0.97)$ & $0.96(0.73-1.26)$ \\
\hline Secondary & $0.76(0.60-0.96)$ & $0.71(0.61-0.83)$ & $0.76(0.61-0.96)$ \\
\hline Higher & $0.71(0.52-0.97)$ & $0.64(0.39-1.03)$ & $0.71(0.53-0.97)$ \\
\hline \multicolumn{4}{|l|}{ Mother's employment status } \\
\hline No (RC) & 1.00 & 1.00 & 1.00 \\
\hline Yes & $1.1(0.99-1.24)$ & $1.03(0.89-1.18)$ & $1.10(0.98-1.23)$ \\
\hline \multicolumn{4}{|l|}{ Father's education level } \\
\hline No education (RC) & 1.00 & 1.00 & 1.00 \\
\hline Primary & $0.85(0.74-0.97)$ & $0.85(0.85-0.85)$ & $0.85(0.75-0.97)$ \\
\hline Secondary & $0.69(0.58-0.82)$ & $0.68(0.57-0.79)$ & $0.70(0.59-0.82)$ \\
\hline Higher & $0.61(0.52-0.70)$ & $0.59(0.51-0.68)$ & $0.61(0.53-0.70)$ \\
\hline \multicolumn{4}{|l|}{ Father's occupation } \\
\hline Professional (RC) & 1.00 & 1.00 & 1.00 \\
\hline Sales & $1.04(0.86-1.25)$ & $1.02(0.86-1.22)$ & $1.03(0.86-1.24)$ \\
\hline Agricultural - self employ & $1.06(0.79-1.43)$ & $1.03(0.99-1.07)$ & $1.06(0.79-1.42)$ \\
\hline Agricultural - employ & $1.04(0.79-1.37)$ & $1.02(0.94-1.11)$ & $1.04(0.79-1.36)$ \\
\hline Services & $1.22(0.98-1.53)$ & $1.24(0.93-1.66)$ & $1.22(0.97-1.52)$ \\
\hline Skilled manual & $1.1(0.82-1.46)$ & $1.08(0.84-1.39)$ & $1.09(0.82-1.45)$ \\
\hline
\end{tabular}


Table 4 Odds Ratio from multilevel generalized linear regression to stunting status of children in Bangladesh, BDHS-2014 (Continued)

\begin{tabular}{|c|c|c|c|}
\hline \multirow[t]{2}{*}{ Variable } & \multicolumn{3}{|c|}{ Odds Ratio (95\% Confidence Interval) } \\
\hline & Model $1^{\text {a }}$ & Model $2^{b}$ & Model $3^{c}$ \\
\hline Household and domestic & $0.72(0.51-1.02)$ & $0.81(0.74-0.89)$ & $0.72(0.50-1.03)$ \\
\hline Don't work & $1.16(0.55-2.43)$ & $1.23(1.09-1.38)$ & $1.16(0.55-2.43)$ \\
\hline \multicolumn{4}{|l|}{ Wealth index } \\
\hline Poorest (RC) & 1.00 & 1.00 & 1.00 \\
\hline Poorer & $0.79(0.63-0.99)$ & $0.80(0.78-0.82)$ & $0.79(0.63-0.99)$ \\
\hline Middle & $0.77(0.70-0.84)$ & $0.78(0.74-0.82)$ & $0.77(0.69-0.85)$ \\
\hline Richer & $0.63(0.49-0.82)$ & $0.64(0.41-1.00)$ & $0.62(0.47-0.82)$ \\
\hline Richest & $0.43(0.32-0.57)$ & $0.45(0.31-0.65)$ & $0.42(0.31-0.57)$ \\
\hline No. of observation & 6900 & 6900 & 6900 \\
\hline AIC & 8191.249 & 8243.575 & 8190.982 \\
\hline $\mathrm{BIC}$ & 8464.82 & 8517.146 & 8471.393 \\
\hline \multicolumn{4}{|l|}{$\begin{array}{l}\text { Intraclass Correlation } \\
\text { Coefficient (ICC) }\end{array}$} \\
\hline Division & 0.0166945 & & 0.0146549 \\
\hline Residence & & 0.0004238 & 0.0187098 \\
\hline \multicolumn{4}{|l|}{$\begin{array}{l}\text { Median Odds } \\
\text { Ratio (MOR) }\end{array}$} \\
\hline Division & 1.252 & & 1.234 \\
\hline Residence & & 1.036 & 1.117 \\
\hline \multicolumn{4}{|l|}{$\begin{array}{l}\text { Random-effects Parameters } \\
\text { Estimate }(95 \% \mathrm{CI})\end{array}$} \\
\hline Residence & & $0.04(0.00-0.57)$ & $0.12(0.04-0.33)$ \\
\hline Division & $0.24(0.13-0.42)$ & & $0.22(0.11-0.44)$ \\
\hline$P$-value & $<0.001$ & 1.00 & $<0.001$ \\
\hline
\end{tabular}

RC stands for 'reference category'

${ }^{a}$ Model 1: two level generalized linear regression considering division as second level hierarchical factor

${ }^{b}$ Model 2: two level generalized linear regression considering residential status as second level hierarchical factor

${ }^{c}$ Model 3: three level generalized linear regression considering residential status as third level and division as second level hierarchical factors

less likely to be stunted than male children $(\mathrm{OR}=0.89$, $95 \% \mathrm{CI}=0.78,1.02)$. Other risk factors which significantly reduced the OR of stunting included children of mothers with normal BMI $(\mathrm{OR}=0.84,95 \% \mathrm{CI}=0.76$, $0.93)$, higher educational level in mother or father $(\mathrm{OR}=$ $0.71,95 \% \mathrm{CI}=0.53,0.97$ and $\mathrm{OR}=0.61,95 \% \mathrm{CI}=0.53$, 0.70 respectively), and children of richest wealth index $(\mathrm{OR}=0.42,95 \% \mathrm{CI}=0.31,0.57)$.

\section{Discussion}

In our multilevel analysis, we have found that children of age group 12-47 months, male, born with preceding birth interval less than 24 months, having underweight mothers, having mothers and fathers with lower education level and of poorest wealth index are more likely to be stunted.

Results from multilevel logistic regression show that as children grow up they become more likely to be stunted. This result is consistent with other studies in Bangladesh $[17,36]$ and also in neighboring countries: India [47],
Nepal [48-50], China [51]. An increasing pattern of stunting by age is found to be undeviating with increasing pattern of communicable childhood diseases by age [52]. As discussed by Hong, Banta and Betancourt [23], this may partly be due to starting other foods along with breastfeeding to a child after 6 months of age, which increases the likelihood of taking polluted foods and minimizes the essential safety provided by breast milk. Moreover, children initiate crawling nearby at this age and are more probable to be carried out-of-doors, which makes them exposure to additional toxicities. Among the under five children, it is most likely to be stunted between age 12-47 months. Therefore, caring at early childhood is more likely to be protective and stunting becomes more prospective as the child becomes more dependent for caloric intake from foods which should be improved. More elaborative researches are needed to demonstrate in this regard.

Male children are found to be insignificantly more stunted than female. Gender has been identified a risk 
factor in many studies [25, 30, 33, 34]. Standard binary logistic regression (single level) also gave insignificant evidence about gender to be determinant of child malnutrition with BDHS-2014 data [17].

Coherent with other studies [26, 30, 33, 34] children of second birth order with less than 24 months birth interval are more likely to be stunted than children of first birth order and children of second birth order with more than 48 months birth interval. The association between stunting and second-order births with shorter interval may be due to competition for food within a household and mother may fail to take care two babies together within limited household.

Similar to our findings some studies confirmed association of child malnutrition with mother's malnutrition [53]. In a traditionally patriarchal society such as Bangladesh, the availability and accessibility of nutritious foods for under-five children is highly dependent on the conduct of maternal duties and responsibilities towards their children which may be impaired when mothers are malnourished.

Education of parents is found to be a preventive factor of child malnutrition which is expected. Education always plays a positive role in health and diseases [37, 39]. Educated parents have more knowledge about health, nutrition, proper child care, health services, hygiene, proper food for children etc. Educated parents also contribute positively to promote the household income which is supportive to provide nutritional diet to their children. Moreover, Islam and his colleagues [54] discussed in this aspect that educated mothers are able to use insufficient family income and offered healthcare services efficiently, can minimize the family size, can sustain improved health stimulating behaviors and can afford better healthcare to their children [55, 56]. All these features may subsidize to keep children in virtuous nourishment.

Nutritional status is called a reflecting indicator of a family's economic condition. This study also shows that children of families with poorest wealth index are most likely to be stunted, as found in other studies conducted in Bangladesh and in other countries [46-50, 57, 58]. This can be attributed to the fact that higher socioeconomic households have more ability to allocate necessary resources regarding nutrition for their children than poorer families. Reasonably allocation of more resources to their children improves their health conditions by reducing multiple health risks.

However, parent's employment status does not found to have any significant role in managing child malnutrition.

Main strength of the study is that the data used in this study is a nationally representative, large sample size and enough information on child malnutrition. The study also has some limitations. The data don't have information on some important factors like amount of diet given to children, mobility pattern of children etc. Some existed variables in the data including size of child at birth, antenatal visits, duration of breast feeding etc. are not included in the model due to a lot of missing information.

\section{Conclusion}

In conclusion, this study clearly reveals that multilevel modeling should be used for hierarchical data to predict significant determinant child malnutrition in correct level. This study also has been identified using multilevel generalized linear regression model that under-five children who are male, of second birth order with less than 24 months, of underweight mothers, of lower educated parents and of poorest family are on high risk to be stunted. Therefore, policy makers and government should pay their attention more to take proper initiative to promote nutritional status in Bangladesh. Government may take necessary steps to aware women of reproductive age about adverse effect of short birth interval. Government and non-government organizations related to the health and nutrition should generate effective program to increase awareness of parents about the standard height and weight according to age and gender of the children. In addition, government may implement child care leave for employed parents with under-five children. Government also may establish childcare center with proper infrastructure, so that employed parents are not obligatory to leave their children at home under illiterate maid. Government may also encourage employed couples to stay with their older parents (or relatives) through some intervention to avoid the same. Overall, necessary steps may be taken to make people educated and to reduce household wealth inequality to improve nutritional status of children.

\section{Abbreviations \\ AARR: Average Annual Rate of Reduction; AIC: Akaike Information Criterion; BDHS: Bangladesh Demographic and Health Survey; BIC: Bayesian Information Criterian; BMI: Body Mass Index; Cl: Confidence Interval; ICC: Intraclass Correlation Coefficient; MOR: Median Odds Ratio; NIPRT: National Institute of Population Research and Training; OR: Odds Ratio; SD: Standard Deviation; WHA: World Health Assembly; WHO: World Health Organization}

\section{Acknowledgements \\ Authors would like to acknowledge Jenny Jung, Yale Fox International Fellow, Yale University, New Haven, CT, United States of America for her constructive feedback to update the paper in English.}

\section{Authors' contributions}

Access of data and material collection: PS. Statistical analysis and interpretation: PS and MMR. Drafting the manuscript: PS, MMR and JA. Contributed to discussion and finishing: PS, MMR and JA. All authors have read and approved the manuscript. 


\section{Availability of data and materials}

Available at: https://dhsprogram.com.

\section{Ethics approval and consent to participate}

Not applicable.

\section{Consent for publication}

Not applicable.

\section{Competing interests}

The authors declare that they have no competing interests.

\section{Author details}

${ }^{1}$ Department of Statistics, University of Rajshahi, Rajshahi, Bangladesh. ${ }^{2} \mathrm{Global}$ Public Health Research Foundation, Dhaka, Bangladesh.

\section{Received: 8 April 2019 Accepted: 30 July 2019}

\section{Published online: 07 October 2019}

\section{References}

1. World Health Organization. [cited 2019 Mar 10]. Available from: https:// www.who.int/topics/malnutrition/en/

2. Pena M, Bacallao J. Malnutrition and poverty. Annu Rev Nutr. 2002:22:24153. https://doi.org/10.1146/annurev.nutr.22.120701.141104.

3. Farhadi S, Ovchinnikov RS. The relationship between nutrition and infectious diseases: a review. Biomed Biotechnol Res J. 2018;2:168-72. https://doi.org/10.4103/bbrj.bbrj_69_18.

4. World Health Organization. [cited 2019 Mar 10]. Available from: https:// www.who.int/news-room/fact-sheets/detail/malnutrition.

5. Nutrition country profiles: Bangladesh. [cited 2019 Mar 10]. Available from: http://www.fao.org/ag/agn/nutrition/bgd_en.stm.

6. Hossain A, Islam S, Munira S, Farzana S, Sarker S, Ahmed R, et al. Infant and child mortality status of Bangladesh: a study on demographic and health survey. Asian J Soc Sci Stud. 2018;3(10):43-53 https://doi.org/10.20849/ajsss.v3i1.330.

7. Bangladesh demographic and health survey (BDHS), 2007. Dhaka: National Institute of Population Research and Training; 2007. p. 346. [cited 2017 Oct 24]. Available from: https://www.unicef.org/bangladesh/BDHS2007_Final.pdf.

8. Bangladesh Demographic and Health Survey (BDHS), 2011. Dhaka: NIPORT, Mitra and Associates, and ICF International; 2013. [cited 2017 Oct 24]. Available from: https://dhsprogram.com/pubs/pdf/fr265/fr265.pdf.

9. de Onis M, Dewey KG, Borghi E, Onyango AW, Blössner M, Daelmans B, et al. The World Health Organization's global target for reducing childhood stunting by 2025: rationale and proposed actions. Matern Child Nutr. 2013;9(2):6-26.

10. Global target 2025. [cited 2017 Oct 24]. Available from: https://www.who. int/nutrition/global-target-2025/en/.

11. Alom J, Quddus MA, Islam MA. Nutritional status of under-five children in Bangladesh: a multilevel analysis. J Biosoc Sci. 2012;44(05):525-35.

12. McGregor GS, Cheung YB, Cueto S, Glewwe P, Richter L, Strupp B. Development potential in the first 5 years for children in developing countries. Lancet. 2007;369(9555):60-70.

13. Faruque ASG, Ahmed AMS, Ahmed T, Islam MM, Hossain MI, Roy SK, et al. Nutrition: basis for healthy children and mothers in Bangladesh. J Health Popul Nutr. 2008;26(3):325-39.

14. Hasan MT, Soares MRJ, Williams GM, Mamun AA. Forecasting the progress towards the target of millennium development goal $1 \mathrm{C}$ in children under 5 years of age in Bangladesh. Public Health Nutr. 2015;18(10):1728-36. https:// doi.org/10.1017/S1368980014003279.

15. Ahmed CN, Hossain T, Mandal MI, Mothabbir BN, Rahman G, Islam M, et al. Community-based management of acute malnutrition in Bangladesh: feasibility and constraints. Food Nutr Bull. 2014;35:277-85.

16. Bairagi R, Chowdhury MK. Socioeconomic and anthropometric status and mortality of young children in rural Bangladesh. Int J Epidemiol. 1994;23: 1179-84 https://doi.org/10.1093/ije/23.6.1179.

17. Das S, Gulshan J. Different forms of malnutrition among under five children in Bangladesh: a cross sectional study on prevalence and determinants. BMC Nutrition. 2017;3:1. https://doi.org/10.1186/s40795-016-0122-2.

18. Das $\mathrm{S}$, Hossain MZ. Levels and determinants of child undernutrition in Bangladesh. Pak J Nutr. 2008;24:301-23.

19. Das $\mathrm{S}$, Hossain MZ, Islam MA. Predictors of child chronic malnutrition in Bangladesh. Proc Pak Acad Sci. 2008:45:137-55.
20. Das S, Rahman RM. Application of ordinal logistic regression analysis in determining risk factors of child malnutrition in Bangladesh. Nutr J. 2011; 10(1):124. https://doi.org/10.1186/1475-2891-10-124.

21. Fakir AMS, Khan MWR. Determinants of malnutrition among urban slum children in Bangladesh. Health Econ Rev. 2015;5:22. https://doi.org/10.11 86/s13561-015-0059-1.

22. Hasan MT, Magalhaes RJS, Williams GM, Mamun AA. The role of maternal education in the 15-year trajectory of malnutrition in children under 5 years of age in Bangladesh. Matern Child Nutr. 2016;12(4):929-39. https://doi. org/10.1111/mcn.12178.

23. Hong R, Banta JE, Betancourt JA. Relationship between household wealth inequality and chronic childhood under-nutrition in Bangladesh. Int J Equity Health. 2006:5:15. https://doi.org/10.1186/1475-9276-5-15.

24. Islam A, Biswas T. Chronic Stunting among under 5 children in Bangladesh a Situation Analysis. Adv Pediatr Res. 2015:2:18 https://doi. org/10.12715/apr.2015.2.18.

25. Islam MS, Jothi JS, Islam M, Huq AKO. Nutritional status of rural and urban under-five children in Tangail District, Bangladesh. Int J Innov Appl Stud. 2014:8(2):841-8.

26. Jesmin A, Yamamoto SS, Malik AA, Haque A. Prevalence and determinants of chronic malnutrition among preschool children: A cross-sectional study in Dhaka City, Bangladesh. J Health Popul Nutr. 2001;29:494-509.

27. Khan REA, Raza MA. Nutritional status of children in Bangladesh: measuring composite index of anthropometric failure (CIAF) and its determinants. Pakistan J Commerce Soc Sci. 2014;8(1):11-23.

28. Mohsena M, Goto R, Taylor CGNM. Socioeconomic and demographic variation in nutritional status of under-five Bangladeshi children and trend over the twelve-year period 1996-2007. J Biosoc Sci. 2017;49(2):222-38. https://doi.org/10.1017/S0021932016000328

29. Mondal D, Minak J, Alam M, Liu Y, Dai J, Korpe $P$, et al. Contribution of enteric infection, altered intestinal barrier function, and maternal malnutrition to infant malnutrition in Bangladesh. Infect Dis Soc Am. 2011;54(2):185-92.

30. Mostafa KS. Socio-economic determinants of severe and moderate stunting among under-five children of rural Bangladesh. Malays J Nutr 2011:17(1):105-18.

31. Muaz SSA, Hasan MR, Shamim SA, Dev A, Kamar S. Nutritional status of 1-5 years children of the tea Workers in Sylhet Division. Bangladesh J Child Health. 2010;34(1):11-6.

32. Rahman A, Biswas SC. Nutritional status of Under-5 children in Bangladesh. S Asian J Popul Health 2009:2(1):1-11.

33. Rahman A, Chowdhury S, Hossain D. Acute malnutrition in Bangladeshi children: levels and determinants. Asia Pac J Public Health. 2009:21(3):294-302

34. Rayhan MI, Khan MSH. Factors causing malnutrition among under five children in Bangladesh. Pak J Nutr. 2006;5(6):558-62.

35. Srinivasan CS, Zanello G, Shankar B. Rural-urban disparities in child nutrition in Bangladesh and Nepal. BMC Public Health. 2013;13:581. https://doi.org/1 0.1186/1471-2458-13-581.

36. Talukder A. Factors associated with malnutrition among under-five children: illustration using Bangladesh demographic and health survey, 2014 data. Children. 2017:4:88. https://doi.org/10.3390/children4100088.

37. Rahman MS, Howlader T, Masud MS, Rahman ML. Association of Low-Birth Weight with Malnutrition in Children under Five Years in Bangladesh: Do Mother's Education, Socio-Economic Status, and Birth Interval Matter? PloS One. 2016;11(6):e0157814. https://doi.org/10.1371/journal.pone.0157814.

38. Ahmed T, Mahfuz M, Ireen S, Ahmed AMS, Rahman S, Islam MM, et al. Nutrition of children and women in Bangladesh: trends and directions for the future. J Health Popul Nutr. 2012;30(1):1-11.

39. Rahman MM, Saima U, Goni MA. Impact of maternal household decisionmaking autonomy on child nutritional status in Bangladesh. Asia Pac Public Health. 2015;27(5):509-20. https://doi.org/10.1177/1010539514568710.

40. Rabbi AMF, Karmaker SC. Determinants of child malnutrition in Bangladesh - a multivariate approach. Asian J Med Sci. 2014;6(2):85-90.

41. Goldstein H. Multilevel Statistical Models. 2nd ed. London: Edward Arnold; 1995.

42. Snijders TAB, Bosker RJ. Multilevel analysis - an introduction to basic and advanced multilevel modeling. Thousand Oaks: CA SAGE Publications; 1999.

43. Akombi BJ, Agho KE, Hall JJ, Merom D, Burt TA, Renzaho AMN. Stunting and severe stunting among children under-5 years in Nigeria: a multilevel analysis. BMC Pediatr. 2017. https://doi.org/10.1186/s12887-016-0770-z.

44. Alemu ZA, Ahemed AA, Yalew AW, Birhanu BS, Zaitchik BF. Individual and community level factors with a significant role in determining child height- 
for-age Z score in east Gojjam zone, Amhara regional state, Ethiopia: a multilevel analysis. Archives of Public Health. 2017. https://doi.org/10.1186/ s13690-017-0193-9.

45. Bangladesh Demographic and Health Survey (BDHS), 2014. Dhaka: NIPORT, Mitra and Associates, and ICF International; 2016. [cited 2017 Oct 24]. Available from: https://dhsprogram.com/pubs/pdf/FR311/FR311.pdf.

46. National Institute of Population Research and Training - NIPORT/Bangladesh, Mitra and Associates/Bangladesh, and ICF International. Bangladesh Demographic and Health Survey 2014. Dhaka: NIPORT, Mitra and Associates, and ICF International; 2016. [cited 2016 Apr 8]. Available from: https:// dhsprogram.com/pubs/pdf/FR311/FR311.pdf.

47. Meshram II, Arlappa N, Balakrishna N, Mallikharjuna Rao K, Laxmaiah A, Brahmam GN. Trends in the prevalence of under-nutrition, nutrient and food intake and predictors of under-nutrition among under five year tribal children in India. Asia Pac J Clin Nutr. 2012;21(4):568-76.

48. Sah N. Determinants of child malnutrition in Nepal: a case analysis from Dhanusha, central Terai of Nepal. J Nepal Health Res Counc. 2004;2(2):50-5.

49. Sapkota VP, Gurung CK. Prevalence and predictors of underweight, stunting and wasting in under-five children. J Nepal Health Res Counc. 2009;7(15):120-6.

50. Ruwali D. Nutritional status of children under five years of age and factors associated in Padampur VDC, Chitwan. Health Prospect. 2011;10:14-8. https://doi.org/10.3126/hprospect.v10i0.5639.

51. Zhai F-Y, Wang H-J, Chang S-Y, Fu D, Ge K, Popkin BM. The current status, trend, and influencing factors to malnutrition of infants and children in China. J Community Nutr. 2004;6(2):78-85.

52. Casa JA, Dachs N, Bambas A. Health disparities in Latin America and the Caribbean: The role of social and economic determinants. Washington DC Pan American Health Organization; 2001. p. 22-49.

53. Khalid N, Aslam Z, Kausar F, Irshad H, Anwer P. Maternal malnutrition and its kick on child growth: an alarming trim for Pakistan. J Food Nutr Popul Health. 2017;1(3):24.

54. Islam MM, Alam M, Tariquzzaman M, Kabir MA, Pervin R, Begum M, Khan $\mathrm{MMH}$. Predictors of the number of under-five malnourished children in Bangladesh: application of the generalized poisson regression model. BMC Public Health, 2013; 13(11). Doi:https://doi.org/10.1186/1471-2458-13-11.

55. Hien NN, Kam S. Nutritional status and the characteristics related to malnutrition in children under five years of age in Nghean, Vietnam. J Prev Med Publ Health. 2008;41 (4):232-40

56. Frost MB, Forste R, Haas DW. Maternal education and child nutritional status in Bolivia: finding the links. Soc Sci Med. 2005;60(2):395-407.

57. Babatunde RO, Olagunju Fl. Prevalence and determinants of malnutrition among under-five children of farming households in Kwara state, Nigeria. J Agric Sci. 2011;3(3):173-81.

58. Sarkar D, Haldar SK. Socioeconomic Determinants of Child Malnutrition in India: Evidence from NFHS-III. 2014. [cited 2017 Oct 24]. Available from: http://ssrn.com/ abstract=2420293 or https://doi.org/10.2139/ssrn.2420293.

\section{Publisher's Note}

Springer Nature remains neutral with regard to jurisdictional claims in published maps and institutional affiliations.

Ready to submit your research? Choose BMC and benefit from:

- fast, convenient online submission

- thorough peer review by experienced researchers in your field

- rapid publication on acceptance

- support for research data, including large and complex data types

- gold Open Access which fosters wider collaboration and increased citations

- maximum visibility for your research: over $100 \mathrm{M}$ website views per year

At BMC, research is always in progress.

Learn more biomedcentral.com/submissions 